\title{
Catalysis: An old but new challenge for graphene-based materials
}

\author{
Ljubisa R. Radovic a,b,*, Camila Mora-Vilches a, Adolfo J. A. Salgado-Casanova a \\ a Department of Chemical Engineering, University of Concepción, Concepción, Chile \\ ${ }^{\mathrm{b}}$ Department of Energy \& Mineral Engineering, Penn State University, University Park, PA, USA
}

\section{A R T I C L E I N F O}

Article history:

Received 5 May 2014

Accepted 7 May 2014

Published 20 June 2014

\section{Keywords:}

Graphene-based material

Challenge

Carbon nanotube

Nanosheet

\begin{abstract}
A B S T R A C T
An assessment is offered regarding the progress made, and the remaining challenges, in the field of carbocatalysis. The fundamental principles that govern the preparation and performance of $\mathrm{sp}^{2}$-hybridized carbon materials in heterogeneous catalysis have been known for decades, and the level of understanding of key issues - especially the importance of textural and ion-exchange properties (i.e., surface area, pore size distribution, and proton transfer) - remains quite satisfactory. The opportunities for novel catalytic materials - especially graphene nanosheets and carbon nanotubes - are tremendous, especially when it comes to taking advantage of their structural order, such that electron transfer can be both better understood and controlled to enhance catalytic activity and selectivity.
\end{abstract}

(C) 2014, Dalian Institute of Chemical Physics, Chinese Academy of Sciences. Published by Elsevier B.V. All rights reserved.

\section{Introduction}

There exists much renewed interest in the catalytic applications of $\mathrm{sp}^{2}$-hybridized (i.e., graphene-based) carbon materials. This is evidenced not only by the exponential growth of relevant publications - as of this writing, ISI's Web of Science lists 13,666 with titles containing 'carbon*' and 'catalys'' and 426 with titles containing 'graphene*' and 'catalys'' -- but also by the continued popularity of the biennial CarboCat conferences (most recently in Bressanone-Brixen, Italy, the next one in 2014 in Trondheim, Norway) and by the half-century-long tradition of catalysis-oriented symposia at annual international conferences on carbon (most recently, in 2013, in Rio de Janeiro, Brazil, the next one in 2014 in Jeju, Korea). Comprehensive $[1,2]$ and updated reviews [3-10] on this subject, which cover both the science and the technology, are readily available, including a dedicated monograph [11]. So our purpose in the present contribution is to highlight and illustrate two fundamental issues related to both relevant processes and products: (1) In the development of new or improved processes, does posterity take advantage of what is well known about existing processes? (2) In the development of novel products, do more recent studies recognize the relevance and comparative (dis)advantages of the more traditional products?

An example, randomly selected from a recent issue of the Carbon journal, will suffice to set the stage for such an approach. "Highly efficient supports" [12] for platinum fuel-cell catalysts (at loadings as high as $20 \mathrm{wt} \%$ ) were recently prepared from graphene oxide (GO, obtained by exfoliation of natural graphite flakes) upon its treatment with ammonium sulfate and subsequent thermal treatment at $235^{\circ} \mathrm{C}$ in argon. The authors did not quantify any of their surface properties; instead, they showed photographic evidence of their changes. Ultrasonication for $12 \mathrm{~h}$ produced a stable dispersion of the hydrophilic GO particles in water, as expected, which was lost due to precipitation upon thermal treatment ( $\mathrm{rGO}$ ) and recovered when thermal treatment was preceded by sulfonation (S-rGO); in the latter sample, the authors could not distinguish how much of the $1.4 \mathrm{mmol} / \mathrm{g}$ of titrated $\mathrm{NaOH}$ corresponded to sulfonate vs oxygen functionalities. The FTIR spectra did sug-

* Corresponding author. Tel: +1-814-8630594; E-mail: lradovic@udec.cl, lrr3@psu.edu 
gest, somewhat surprisingly (given the relatively low reduction temperature used), that there was "a simultaneous reduction of GO in the process of sulfonation", even though the authors did not identify the most prominent $1626 \mathrm{~cm}^{-1}$ band (due perhaps to "polarized $\mathrm{C}=\mathrm{C}$ bond vibration near chemisorbed oxygen" [13]?). What is most puzzling, however, is the finding that "Pt [nanoparticles] are well dispersed on S-rGO ... with diameters in the $2.5 \mathrm{~nm}$ range" and also that "well dispersed Pt NPs are found on GO surfaces because of the presence of abundant functional groups". The discussion offered by the authors is superficial on several scores, especially because they do not compare these results to the very abundant literature on carbon-supported Pt catalysts [1]. First, it is well known that the presence of acidic surface functionalities typically does not result in high catalyst dispersion when chloroplatinic acid is used, because of repulsion of $\mathrm{PtCl}_{6}{ }^{2-}$ anions by the (typically) negatively charged carbon surface. (The authors did not report either the point of zero charge of their supports or the $\mathrm{pH}$ of their ethylene-glycol-containing suspension but, for example and as expected, Watkins et al. [14] reported that commercially available "sulfonate-functionalized carbon nanoparticles ... possess negatively charged sulfonate functional groups".) Furthermore, even though sulfonated carbons have been the subject of much recent research [13,15-17], their surface chemical properties $[18,19]$ have yet to be fully characterized and the presumed virtue of "sulfonic groups that can immobilize the $\mathrm{Pt}$ NPs and avoid their aggregation on S-rGO" remains to be confirmed and compared, say, to a similar role of CO-yielding oxygen functional groups $[1,20]$. Finally, the authors reported that the electrochemical surface area order was Pt/S-rGO (82.6 $\mathrm{m}^{2} / \mathrm{g}$ ) > Pt/GO (61.8) > Pt/rGO (55.6) > Pt/C (Johnson-Matthey commercial catalyst, 53.1), which agreed with the electrocatalytic activity order (at $20 \mathrm{wt} \% \mathrm{Pt}$ ), Pt/S-rGO (12.6 A/g) > Pt/GO (7.5) > Pt/rGO (5.8) > Pt/C (4.9); it is puzzling, however, that they attribute it to "the optimized dispersion and size distribution of Pt NPs and the enhanced [triple phase boundary] by means of the function of $-\mathrm{SO}_{3} \mathrm{H}$ groups grafted in $\mathrm{rGO}^{\prime}$ (see section on proton transfer below).

It is somewhat disappointing that too many reports such as the one analyzed above, and even recent reviews dealing with carbon catalysis -- and there have been quite a few, mostly devoted to the virtues of 'nanocarbons' - have not been more comprehensive. Because the properties that are well known to be responsible for the "unparalleled flexibility in tailoring carbon's properties to specific needs..." [1] - a phrase often quoted $[5,13,21]$ but too often superficially invoked - have been clearly summarized more than a decade ago [1]: wide range of tailorable surface area and pore size distribution, surface charge ranging from quite positive (at relatively low suspension $\mathrm{pH}$ ) to very much negative (at high $\mathrm{pH}$ ), and inertness/reducibility/ conductivity that can be adjusted by judicious selection or design of carbon crystallinity and surface functionality. Certainly the importance of physical surface (or textural) properties, as well as of those chemical surface properties that affect proton transfer, has been understood for several decades $[1,6,10,11,18,22]$. Whether this has resulted in successful recipes for the development of nanocarbon-based catalysts, espe- cially taking into account the undoubtedly important but still insufficiently understood electron transfer, is discussed below. Given the existence of an almost overwhelming body of literature, the approach adopted here consists in analyzing the findings of illustrative publications mentioned in some of the recent reviews of carbon (i.e., graphene-based) materials in catalysis.

\section{Importance of textural properties}

In a review of 'carbocatalysis', among the virtues of graphene-based materials as catalysts in their own right, Dreyer et al. [7] remind the reader that "[h]igh surface area carbons are particularly useful ... because of their high ratio of catalytically active surface area to weight". It is interesting that they mention carbon molecular sieves "with exceptionally high surface areas, reaching over $3000 \mathrm{~m}^{2} \mathrm{~g}^{-1}$ in some instances", but not the much more ubiquitous activated carbons, whose tailoring of textural properties is a well documented science. While they clearly misrepresent the virtues of graphite (" $[\mathrm{b}]$ eyond its high surface area and unique chemical properties"), they leave the reader unnecessarily confused about the relative roles of surface 'physics' vs 'chemistry' in the utility of carbons in synthetic chemistry. The relevant issues have been well established for decades: the textural properties (surface area and pore size distribution) are more important when carbons are used as catalyst supports, especially at high catalyst loadings and for large adsorbing/reacting molecules. When carbons are used as catalysts, as evidenced by the effectiveness of materials with both very low $\left(<1 \mathrm{~m}^{2} / \mathrm{g}\right)$ and very high surface area $(>1000$ $\mathrm{m}^{2} / \mathrm{g}$ ), the proton- and/or electron-transfer properties are more important (see next sections).

The potentially genuine importance of textural properties has been invoked, for example, by suggesting [23] that carbon nanotubes (CNT) "show much higher catalyst loading efficiency" than carbon blacks (CB). These authors surmised, however, that "undesired bundling and incomplete functionalization normally only afford limited accessible area and defects" and thus went on to utilize graphene nanoribbons (GNR) which, presumably, "can display much larger surface area" than multiwall CNTs. Their reported order of electrocatalytic activity was indeed Pt/GNR (5.8 A/m²) > Pt/MWCNT (3.3) > Pt/CB (2.4), but they did not discuss the often very complex relationship between the catalytically active surface area and the support surface area, nor did they report measurements of total or accessible areas of their supports. In a similar and ambitiously titled study, Antolini [9] invokes similar arguments, acknowledges that in practice these surface area advantages may be nonexistent (" $s]$ urface area measurement of the reduced [graphene oxide] sheets via nitrogen gas $\mathrm{a}[d]$ sorption yielded a BET value of $466 \mathrm{~m}^{2} / \mathrm{g}$ ") but insists that the "very high surface area of [graphene nanosheets] allows to load high amounts of catalysts maintaining a low metal particle size". Clearly, claims of such advantages of graphenes, and to some extent of nanotubes as well, will remain largely hypothetical in the surprisingly frequent absence of even a rudimentary characterization of their textural properties. 


\section{Importance of proton transfer}

In the above-cited review of Antolini [9], the well established relative importance of surface physics and surface chemistry of carbon materials has arguably been obscured. For example, in a discussion that mimics similar arguments summarized more than a decade ago, the author reminds the reader of the decades-old concept that the "very high surface area of [graphene nanosheets]", as well as that of, say, activated carbons, "allows to load high amounts of catalysts maintaining a low metal particle size, particularly for functionalized GNS" (emphasis added). In support of this statement, the author echoes the findings of Lee et al. [24] by saying that the "difference in [catalyst] particle dispersion and size was explained by the different specific surface area[s] of carbon support materials" [9]. However, Lee et al. [24] provided no measurements of surface area. Instead they refer the reader to a study by another research group [25], in which only different carbon blacks (of unspecified surface chemical properties), rather than GNS or GNR or CNT samples, were compared; furthermore, the well known general correlation between their surface area and catalytic activity (in methanol oxidation) does not necessarily imply a cause-effect relationship. These authors used ethanol-solutions of $\mathrm{Pt}\left(\mathrm{NH}_{3}\right)_{2}\left(\mathrm{NO}_{2}\right)_{2}$ and $\mathrm{RuNO}\left(\mathrm{NO}_{3}\right)_{x}$ in a co-impregnation method and did not analyze the associated proton-transfer effects, i.e., the possibility of specific interactions between these (cationic?) precursors and the (positively?) charged carbon surfaces. In the same paragraph of his review, Antolini [9] also reminds the reader of the well established fact $[1,20]$ that "surface groups on [functionalized graphene nanosheets] may function as anchoring sites for Pt precursor to prevent the aggregation of the Pt nanoparticles". A study by Choi et al. [26] is cited to confirm this in which, indeed, these authors concluded that the "surface functional groups that were formed during GNSs preparation from graphite and the large surface area of the GNSs could be responsible for such small, highly concentrated, and uniformly dispersed Pt nanoparticles". Their use of the word 'could' is of course puzzling, because their research objective should have been not to propose, but to confirm whether these arguments, extensively demonstrated to be valid for, say, activated carbons are also applicable to graphene, as expected. Being apparently oblivious to the 'rules' of proton transfer in graphene-based materials $[1,18,19]$, the authors prepared their (electro)catalysts by mixing hexachloroplatinic acid hydrate with GNS, but their characterization of oxygenated functional groups did not include the crucial analysis of the electrokinetic properties of the catalyst supports, nor did they report the $\mathrm{pH}$ evolution of the resulting suspension; without such knowledge, it cannot be convincingly argued how the "presence of functional groups ... in significant amounts could help the formation of ... uniformly dispersed $\mathrm{Pt}$ nanoparticles at high Pt loadings" [26], especially if the reported BET surface area ( $\left.487 \mathrm{~m}^{2} / \mathrm{g}\right)$ is not particularly large.

It has recently been recalled [4] that the "main influence of graphene [on catalytic activity] is through its ability to donate or withdraw electron density" from metallic or semiconducting (nano)particles supported on it. Thus, Kamat [27] reminded the reader of a century-old "major hurdle" to "use attached catalyst particles with good dispersity" and reported the results of the "use of the two-dimensional morphology of graphene oxide sheets to anchor metal and semiconductor nanoparticles" which presumably offers "a simple and attractive approach toward designing next-generation catalyst systems". Intriguingly, however, the author mentions only in passing that inorganic oxides such as $\mathrm{TiO}_{2}$ or $\mathrm{ZnO}$ "are capable of interacting with graphene oxide via carboxylic acid functional groups". For key details, the reader is referred instead to two of his own recent studies, even though this is a well known catalyst preparation strategy: its scientific basis has been understood quite well for several decades and it must be demonstrated that simple "mixing of two suspensions of $\mathrm{GO}$ and $\mathrm{TiO}_{2}$ (or $\mathrm{ZnO}$ ) results in the binding of oxide particles to graphene oxide flakes" [27]. Neither of these studies [28,29], however, offers any evidence of the role of carboxyl groups, located at graphene edges, in producing a "dispersion of graphene oxide sheets coated with the $\mathrm{TiO}_{2}$ nanoparticles" [29]; such a role is well known to be based on ion exchange (i.e., proton transfer) properties of these groups, which result in electrostatic catalyst/support attraction or repulsion, and are dependent on the point of zero charge of the support and the charge of the catalyst (or catalyst precursor). The implications of these issues for electron transfer are analyzed below.

\section{Importance of electron transfer}

Progress on this front seems to be most difficult, even though it has been more than half a century since a theory that competes with the ubiquitous oxygen transfer mechanism was proposed by Long and Sykes [30] in the context of carbon oxidation [31]. This is illustrated in Fig. 1: upon oxygen adsorption, a catalyst induces the distribution of $\pi$-electrons shown on the right, i.e., by accepting electrons from the graphene layer (e.g., into unfilled bands of a transition metal), the catalyst facilitates both reactant adsorption (by increasing the $\mathrm{C}=0$ bond strength) and product desorption (by decreasing the strength of adjacent $\mathrm{C}-\mathrm{C}$ bonds).

The direction of electron transfer, and the related Fermi-level alteration, is thus the key issue. For example, the electron-donating potassium lowers the work function of graphite and is indeed expected to catalyze $\mathrm{O}_{2}$ dissociation [32]. In an already mentioned study, Kamat and coworkers [29] used "luminescence quenching of the green emissions of $\mathrm{ZnO} \ldots$ as a probe to monitor the electron transfer from excited $\mathrm{ZnO}$ to graphene oxide". The much-investigated role of substitutional boron is another emblematic case [31]: it affects the electron density distribution by (i) reducing the total electron density in

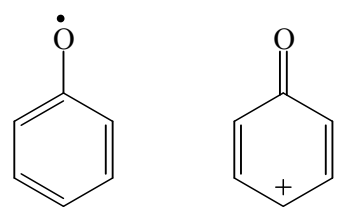

Fig. 1. Postulated electron distribution in graphene in the presence of a catalyst that exerts an electronic effect (adapted from Ref. [30]). 
its vicinity, (ii) decreasing the contribution of delocalized $\pi$-electrons to the electron density on the remaining carbon atoms, and (iii) inducing $\pi$-electron localization on $\mathrm{C}$ atoms due to their higher electronegativity with respect to $B$. The review by Haag et al. [4] also emphasizes the "tunable electrical properties" of graphene and graphene oxide. In particular, they briefly analyzed the properties and performance of $\mathrm{N}$-doped graphene catalysts and noted the obvious facts that "[n]itrogen incorporation into the graphene structure changes the spin density and charge distribution of the surrounding carbon atoms and widens the band gap"; however, they did not provide the essential details and were thus not able to link these changes to either the catalytic activity or the concentration of catalytically active sites. (The fascinating role of $\mathrm{N}$-functionalities or $\mathrm{N}$-substitution in the oxygen reduction reaction is a very popular case in point, but it is beyond the scope of this mini-review.)

$\mathrm{Su}$ and coworkers [6] devote separate sections of their informative review to "electron-transfer-induced changes in the properties of supported nanoparticles" and "defect-related catalytic reactivity". In the summary they note that, even though "conclusive evidence on the nature of [active] sites and their catalytic reactivity is still missing ... large progress in this direction has been definitively made in the last years". As evidence for such progress, for example, they provide a list of "aspects in the design of nanocarbon-semiconductor hybrid materials ... that allow the development of materials with potential[ly] higher performances". Among these, they highlight "[c]arbon nanodots and other carbon surface species [that] may act as efficient solid-state sensitizers to promote visible light absorption"; it is intriguing that they do not provide specific examples, especially in the light of steadily accumulating evidence for photoluminescence behavior of graphene-based materials in the absence of inorganic semiconductors. Thus, for example, Pan and coworkers [33,34] proposed that the origin of photoluminescence excitation (PLE) is "reversible protonation of carbene-like zigzag sites" [33], because the presence of such sites is consistent with the requirements of relatively large HOMO-LUMO gaps, Lewis base character and singly occupied orbitals [34]. Similar arguments regarding the origin of luminescence of graphene quantum dots [35] were invoked by Gupta et al. [36] and Shen et al. [37]. When it comes to defects, it is intriguing that this review highlights a study that apparently focuses on the "high reactivity of the graphene border and basal plane to anchor metal nanoparticles" [6], rather than the role of heteroatom doping or, say, of vacancies, with concomitant and potentially profound changes in electron density distribution. What Moldovan et al. [38] have actually accomplished is confirm, albeit in an elegant study of few-layer graphene, the behavior of graphite that had been amply documented, and qualitatively explained, as early as half a century ago [39-41]: "[s]pherical Pt [nanoparticles] with average sizes of $2 \mathrm{~nm}$ located preferentially at the support topographical defects (e.g., steps and edges) diffuse and coalesce along these defects such that, after annealing to $700{ }^{\circ} \mathrm{C}$, the nanoparticles were located exclusively here" [38].

Over the past decades, computational quantum chemistry has come of age and it is ideally suited to shed additional light on these complex issues. Thus, for example, the study by Boukhvalov and coworkers [42] highlights the mechanistic explorations of catalytic properties of graphene oxide using the methodology of density functional theory (DFT): the authors propose an important role of graphene's neighboring epoxide groups (in the basal plane) in forming diols and thus catalyzing the oxidation of benzyl alcohol to benzaldehyde. Figures 2 and 3 summarize our incipient efforts in this direction [43,44]; our focus is on graphene edges rather than the basal plane, and we

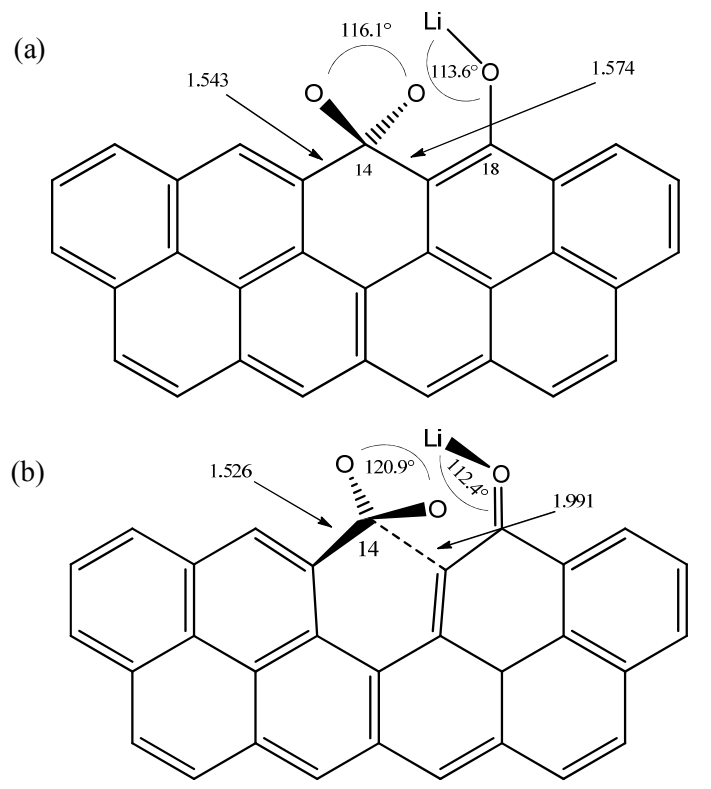

Fig. 3. Adsorption of $\mathrm{O}_{2}$ on an active site adjacent to lithium phenolate in graphene. (a) Intermediate triplet ground state; (b) Triplet transition state.
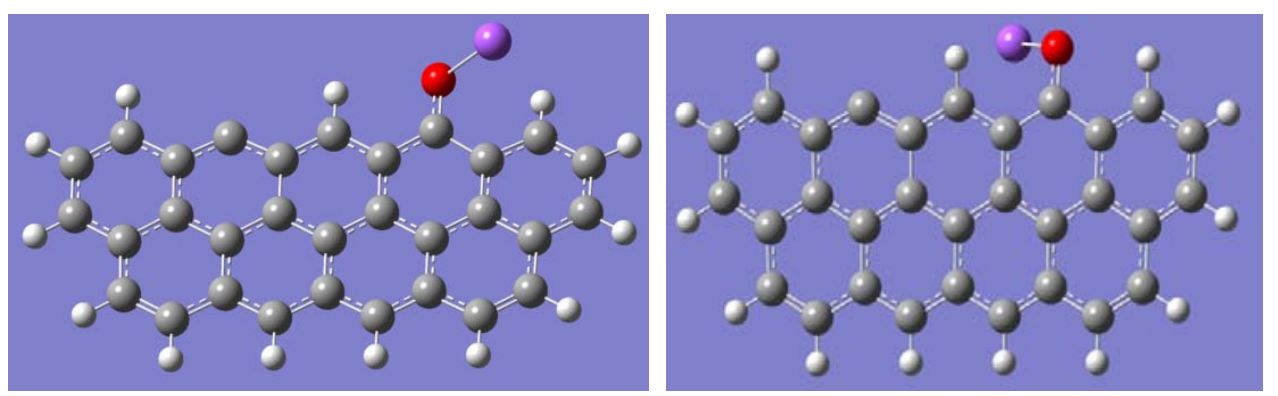

Fig. 2. Optimized geometries of graphenes $\mathrm{C}_{31} \mathrm{H}_{13} \mathrm{ONa}$. Left ( -1426.71469 hartrees): $\mathrm{C}-\mathrm{O}-\mathrm{Na}=99.4^{\circ}, \mathrm{Na}-\mathrm{C}^{*}=0.68 \mathrm{~nm}$; Right $(-1426.71947$ hartrees $)$ : $\mathrm{C}-\mathrm{O}-\mathrm{Na}=101^{\circ}, \mathrm{Na}-\mathrm{C}^{*}=0.43 \mathrm{~nm}$. 
thus performed DFT calculations using representative 2D clusters (or molecules) instead of periodic boundary conditions. The geometric flexibility of the alkali phenolate group is quite remarkable and appears to have a profound effect on electron density distribution: the difference in energy between the two ground states in Fig. 2 (both triplets), whose reactive site 'accessibility' is expected to be drastically different, is only 3.0 $\mathrm{kcal} / \mathrm{mol}$. Such results suggests the possibility of remote control catalysis [45]. In Fig. 3 we illustrate a key portion of a reaction path in alkali-graphene interaction, analogous to that of $\mathrm{O}_{2}$ interaction with a metal-free graphene; the rearrangement is $6.8 \mathrm{kcal} / \mathrm{mol}$ uphill and $\mathrm{CO}_{2}$ is eventually formed upon consecutive rupture of the $\mathrm{C}-\mathrm{C} 14-\mathrm{C}$ bonds.

\section{Summary}

Over the past few decades we have witnessed a tremendous explosion of interest in, and research activity on, the fascinating properties of novel graphene-based materials, including their potential utilization in (electro)catalytic applications. In this excitement about undeniably expanding opportunities, however, there is too much evidence of monologue-like attempts to '(re)invent' even the basic scientific principles that are expected to determine their physico-chemical surface properties and thus govern their catalytic behavior. This applies especially to the roles of textural properties and proton transfer, which should be very well understood based on knowledge of 'conventional' graphene-based materials such as activated carbons and carbon blacks. When it comes to electron transfer - where the structurally much better defined graphene and carbon nanotubes offer greater opportunities for research progress than, say, the more disordered activated carbons or carbon blacks - there is some evidence of new insights. Both the "unique graphitized basal plane structure" [9] and the extensively investigated heteroatom incorporation (not only 0-edge functionalization, but also N-, B-, and P-doping) have been subjected to increasingly detailed experimental and theoretical scrutiny. The extent and direction of charge transfer and its catalytic implications appear to be dependent on both shortand long-range perturbations of electron density and they should be the main focus of carbocatalysis research in the coming years.

\section{Acknowledgments}

Financial support for this study was provided by CONICYT-Chile (Projects FONDECYT 1120609 and PFB-27 CCTE-UDT). Encouragement from Prof. Dangsheng Su is greatly appreciated.

\section{References}

[1] Radovic L R, Rodríguez-Reinoso F. Chem Phys Carbon, 1997, 25: 243

[2] Radovic L R, Sudhakar C. In: Marsh H, Heintz E A, RodríguezReinoso $\mathrm{F}$ eds. Introduction to Carbon Technologies. Alicante, Spain: University of Alicante Press, 1997

[3] Su D S, Perathoner S, Centi G. Catal Today, 2012, 186: 1

[4] Haag D R, Kung H H. Top Catal, 2014, 57: 762

[5] Rodríguez-Reinoso F. Carbon, 1998, 36: 159

[6] Su D S, Perathoner S, Centi G. Chem Rev, 2013, 113: 5782

[7] Dreyer D R, Bielawski C W. Chem Sci, 2011, 2: 1233

[8] Machado B F, Serp P. Catal Sci Technol, 2012, 2: 54

[9] Antolini E. Appl Catal B, 2012, 123-124: 52

[10] Zhu J, Holmen A, Chen D. ChemCatChem, 2013, 5: 378

[11] Serp P, Figueiredo J L (Ed). Carbon Materials for Catalysis. Hoboken: Wiley, 2009

[12] He D P, Kou Z K, Xiong Y L, Cheng K, Chen X, Pan M, Mu S C. Carbon, 2014, 66: 312

[13] Peng F, Zhang L, Wang H J, Lü P, Yu H. Carbon, 2005, 43: 2405

[14] Watkins J D, Lawrence R, Taylor J E, Bull S D, Nelson G W, Foord J S, Wolverson D, Rassaei L, Evans N D M, Gascon S A, Marken F. Phys Chem Chem Phys, 2010, 12: 4872

[15] Barroso-Bujans F, Verdejo R, Arroyo M, Lopez-Gonzalez M D, Riande E, Lopez-Manchado M A. Macromol Rapid Commun, 2008, 29: 234

[16] Barroso-Bujans F, Fierro J L G, Rojas S, Sanchez-Cortes S, Arroyo M, Lopez-Manchado M A. Carbon, 2007, 45: 1669

[17] Mo X H, Lopez D E, Suwannakarn K, Liu Y J, Lotero E, Goodwin J G Jr, Lu C Q.J Catal, 2008, 254: 332

[18] Leon y Leon C A, Radovic L R. Chem Phys Carbon, 1994, 24: 213

[19] Boehm H P. Carbon, 1994, 32: 759

[20] Prado-Burguete C, Linares-Solano A, Rodriguez-Reinoso F, Salinas-Martinez de Lecea C. J Catal, 1989, 115: 98

[21] van Steen E, Prinsloo F F. Catal Today, 2002, 71: 327

[22] Rodriguez-Reinoso F, Linares-Solano A. Chem Phys Carbon, 1989, 21: 1

[23] Wang C N, Li H, Zhao J H, Zhu Y, Yuan W Z, Zhang Y M. Int J Hydrog Energy, 2013, 38: 13230

\section{Graphical Abstract}

\section{Chin. J. Catal., 2014, 35: 792-797 doi: 10.1016/S1872-2067(14)60130-3}

\section{Catalysis: An old but new challenge for graphene-based materials}

Ljubisa R. Radovic* , Camila Mora-Vilches, Adolfo J. A. Salgado-Casanova University of Concepción, Chile; Penn State University, USA

Opportunities for novel catalytic materials - especially graphene nanosheets and carbon nanotubes - are tremendous: electron transfer processes, such as the one illustrated, can be both better understood and controlled to enhance catalytic activity and selectivity.

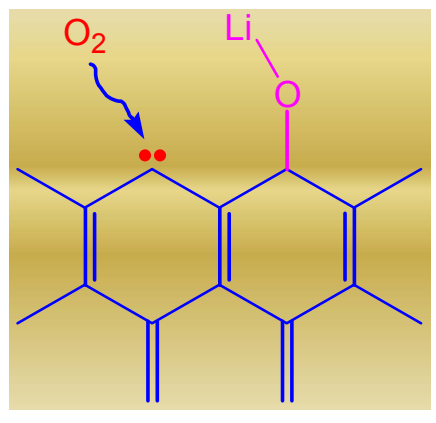


[24] Lee S H, Kakati N, Jee S H, Maiti J, Yoon Y S. Mater Lett, 2011, 65: 3281

[25] Takasu Y, Kawaguchi T, Sugimoto W, Murakami Y. Electrochim Acta, 2003, 48: 3861

[26] Choi S M, Seo M H, Kim H J, Kim W B. Carbon, 2011, 49: 904

[27] Kamat P V.J Phys Chem Lett, 2010, 1: 520

[28] Williams G, Kamat P V. Langmuir, 2009, 25: 13869

[29] Williams G, Seger B, Kamat P V. ACS Nano, 2008, 2: 1487

[30] Long F J, Sykes K W. J Chim Phys, 1950, 47: 361

[31] Radovic L R, Karra M, Skokova K, Thrower P A. Carbon, 1998, 36: 1841

[32] Janiak C, Hoffmann R, Sjövall P, Kasemo B. Langmuir, 1993, 9: 3427

[33] Pan D Y, Zhang J C, Li Z, Wu M H. Adv Mater, 2010, 22: 734

[34] Pan D Y, Zhang J C, Li Z, Wu C, Yan X M, Wu M H. Chem Commun, 2010, 46: 3681

[35] Radovic L R. In: Eder D, Schlögl R eds. Nanocarbon-Inorganic Hybrids: Next Generation Composites for Sustainable Energy Appli- cations. Berlin: Walter De Gruyter, 2014

[36] Gupta V, Chaudhary N, Srivastava R, Sharma G D, Bhardwaj R, Chand S. J Am Chem Soc, 2011, 133: 9960

[37] Shen J H, Zhu Y H, Chen C, Yang X L, Li C Z. Chem Commun, 2011, 47: 2580

[38] Moldovan M S, Bulou H, Dappe Y J, Janowska I, Begin D, Pham-Huu C, Ersen 0.J Phys Chem C, 2012, 116: 9274

[39] Baker R T K. J Adhesion, 1995, 52: 13

[40] Hennig G R. Chem Phys Carbon, 1966, 2: 1

[41] Thomas J M. Chem Phys Carbon, 1965, 1: 121

[42] Boukhvalov D W, Dreyer D R, Bielawski C W, Son Y W. ChemCatChem, 2012, 4: 1844

[43] Salgado A, Radovic L R. In: Proceedings of Carbon 2014 (International Carbon Conference). Jeju, Korea, 2014

[44] Mora-Vilches C, Buljan A, Radovic L R. In: Proceedings of Carbon 2013 (International Carbon Conference). Rio de Janeiro, Brazil, 2013

[45] Delmon B, Froment G F. Catal Rev-Sci Eng, 1996, 38: 69 